\title{
When genotype is not predictive of phenotype: implications for genetic counseling based on 21,594 chromosomal microarray analysis examinations
}

\author{
Idit Maya, MD ${ }^{1,2}$, Reuven Sharony, $\mathrm{MD}^{2,3,4}$, Shiri Yacobson, MSc ${ }^{1}$, Sarit Kahana, $\mathrm{PhD}^{1}$, \\ Josepha Yeshaya, $\mathrm{PhD}^{1}$, Tamar Tenne, $\mathrm{PhD}^{3}$, Ifaat Agmon-Fishman, MSc ${ }^{1}$, Lital Cohen-Vig, $\mathrm{MD}^{1}$, \\ Yael Goldberg, MD ${ }^{1,5}$, Racheli Berger, $\mathrm{PhD}^{5}$, Lina Basel-Salmon, MD, PhD ${ }^{1,2,6,7}$ and \\ Mordechai Shohat, MD $2,5,8$
}

\begin{abstract}
Purpose: To compare the frequency of copy-number variants (CNVs) of variable penetrance in low-risk and high-risk prenatal samples and postnatal samples.

Methods: Two cohorts were categorized according to chromosomal microarray analysis (CMA) indication: group I, low-risk prenatal-women with uneventful pregnancy (control group); group II, high-risk prenatal-women whose fetuses had congenital malformations; and group III, postnatal-individuals with unexplained developmental delay/intellectual disability, autism spectrum disorders, or multiple congenital anomalies. CNVs were categorized based on clinical penetrance: (i) high $(>40 \%)$, (ii) moderate (10-40\%), and (iii) low (<10\%).
\end{abstract}

Results: From 2013 to 2016, 21,594 CMAs were performed. The frequency of high-penetrance CNVs was $0.1 \%(21 / 15,215)$ in group
I, $0.9 \%(26 / 2,791)$ in group II, and $2.6 \%(92 / 3,588)$ in group III. Moderate-penetrance CNV frequency was $0.3 \%$ (47/15,215), $0.6 \%$ $(19 / 2,791)$, and $1.2 \%(46 / 3,588)$, respectively. These differences were statistically significant. The frequency of low-penetrance CNVs was not significantly different among groups: $0.6 \%$ $(85 / 15,215), 0.9 \%(25 / 2,791)$, and $1.0 \%(35 / 3,588)$, respectively.

Conclusion: High-penetrance CNVs might be a major factor in the overall heritability of developmental, intellectual, and structural anomalies. Low-penetrance $\mathrm{CNV}$ alone does not seem to contribute to these anomalies. These data may assist pre- and posttest CMA counseling.

Genet Med advance online publication 20 July 2017

Key Words: chromosomal microarray analysis (CMA); copynumber variation $(\mathrm{CNV})$; penetrance; prenatal diagnosis

\section{INTRODUCTION}

Chromosomal microarray analysis (CMA) is currently recommended as a first-tier test for individuals with unexplained developmental delay/intellectual disability, autism spectrum disorders, or multiple congenital anomalies. ${ }^{1}$ Six percent of pregnancies that resulted in structural malformations and normal karyotype had pathologic copy-number variations (CNVs). Moreover, pregnant women without medical indication for CMA (advanced maternal age) or positive maternal serum screening for Down syndrome who had normal fetal karyotype in prenatal studies were found to have $1.7 \%$ residual risk for pathogenic CMA. ${ }^{2}$ Nevertheless, identical pathogenic CNVs, even among members of the same family, can have different clinical outcomes. ${ }^{3-6}$ This phenomenon can be partially explained by incomplete penetrance. Additional factors might be involved in this process, such as the effect of other CNVs, single-nucleotide variants, and environmental or epigenetic factors. ${ }^{4,7}$ The fact that many CNVs have incomplete penetrance poses a challenge for genetic counseling. ${ }^{9}$ Prenatal counseling in these cases is complicated and knowledge of the penetrance level could help provide more informative counseling. Previous publications estimated the penetrance of common $\mathrm{CNVs}^{5,6,10}$ and specifically for mental and neurological disorders. ${ }^{11-13}$ Since most of the information regarding clinical presentation is from postnatal CMA tests, some of the assumptions and data used were questioned. ${ }^{14}$ This study analyzed the penetrance of 20 known CNVs in prenatal and postnatal samples in order to suggest more informative counseling for these complicated cases.

\section{MATERIALS AND METHODS}

All CMAs were performed by two of the largest laboratories in Israel, both serving similar population groups. Whole-genome approach CMA, using single-nucleotide polymorphism (SNP)-based array platforms, was performed (Illumina, San Diego, CA) (Supplementary Materials and Methods online).

\footnotetext{
${ }^{1}$ Recanati Genetics Institute, Beilinson Hospital, Rabin Medical Center, Petach Tikva, Israel; ${ }^{2}$ Sackler Faculty of Medicine, Tel Aviv University, Tel Aviv, Israel; ${ }^{3}$ The Genetics Institute, Meir Medical Center, Kfar Saba, Israel; ${ }^{4}$ Department of Obstetrics and Gynecology, Meir Medical Center, Kfar Saba, Israel; ${ }^{5}$ Maccabi Health Services, Rehovot, Israel; ${ }^{6}$ Pediatric Genetics Unit, Schneider Children's Medical Center of Israel, Petah Tikva, Israel; ${ }^{7}$ Felsenstein Medical Research Center, Rabin Medical Center, Petah Tikva, Israel;

${ }^{8}$ Bioinformatics Unit, Sheba Cancer Research Center, Sheba Medical Center, Tel Hashomer, Israel. Correspondence: Reuven Sharony (reuvensharony@gmail.com)

The first two authors contributed equally to this work.

The last two authors contributed equally to this work.

Submitted 23 February 2017; accepted 2 May 2017; advance online publication 20 July 2017. doi:10.1038/gim.2017.89
} 


\section{Cohorts}

CMA results from each laboratory were analyzed separately. The two cohorts consisted of 10,184 and 11,410 cases individuals, respectively, recruited from several genetic institutes. However, since the two cohorts were not statistically different from each other, we combine the cohorts for all analyses (Table 1). All cases were unrelated. Inclusion criteria were known specific indication and available CMA results. The cases were divided into three groups: group I, low-risk prenatal-women with uneventful (low-risk) pregnancy (the control group); group II, high-risk prenatal-women whose fetuses had structural malformations detected by prenatal ultrasound scan or magnetic resonance imaging studies (high-risk prenatally); and group III, postnatal- patients with intellectual disability, autism, other cognitive impairment and/or congenital malformation.

We focused on 20 recurrent pathogenic autosomal CNVs detected by CMA with documented penetrance. The two cohorts combined were divided into three categories based on the associated penetrance of these CNVs as reported in the literature (Supplementary Materials and Methods: Tables S1-4).

The prenatal and postnatal frequencies of CMA findings were compared in each penetrance category. The frequencies of these groups were compared to the low-risk group assuming they all have similar variance. A chi-square test was used for comparisons, and $P<0.05$ was considered statistically significant (Table 1). The study was approved by the institutional review board (Beilinson Medical Center).

\section{RESULTS}

From 2013 through 2016, 21,594 CMA tests were performed on 15,215 low-risk pregnancies, 2,791 high-risk pregnancies, and 3,588 postnatal samples. Most of the prenatal CMAs were on DNA extracted from amniotic fluid. Chorionic villus sampling, before anatomic ultrasound of the fetus, was performed in fewer than $10 \%$ of the cases and was evenly distributed between groups I and II. All cases of postnatal CMA used DNA that was extracted from blood.

The first cohort consisted of 10,184 cases. Twenty recurrent CNV were detected in 55/5,149 cases $(1.1 \%)$ in the low-risk prenatal group, in $56 / 2,447$ cases $(2.3 \%)$ in the high-risk prenatal group, and in $129 / 2,588$ cases (5.0\%) postnatally. The second cohort consisted of 11,410 cases. The same 20 recurrent CNVs were detected in 98/10,066 (1.0\%) among the low prenatal risk group, in $14 / 344(4.1 \%)$ in the high prenatal risk group, and in 44/1,000 (4.4\%) postnatally. Penetrance category and the indication for CMA group were compared using the total number (Table 1).

\section{High-penetrance CNV syndromes}

High-penetrance CNVs were detected in 21/15,215 (0.1\%) control cases (low-risk prenatal), 26/2,791 (0.9\%) high-risk prenatal cases, and 92/3,588 (2.6\%) postnatal cases. The difference was statistically significant $(P<0.005)$.

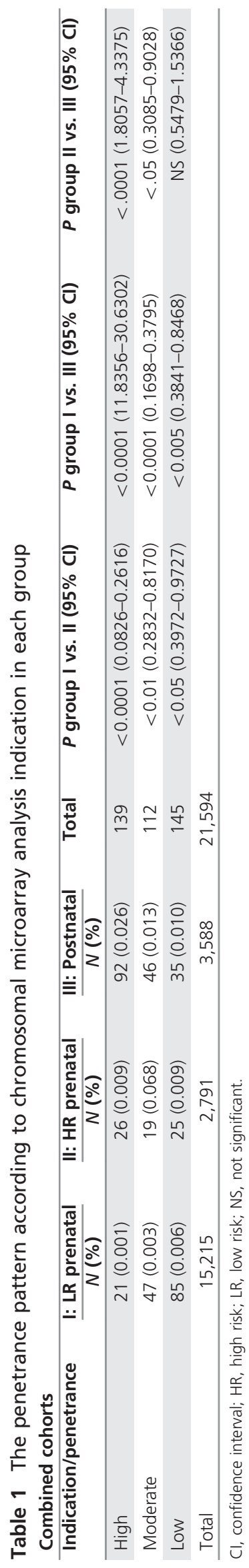




\section{Medium-penetrance CNV syndromes}

Medium-penetrance CNVs were detected in 47/15,215 (0.3\%) control cases (low-risk prenatal), 19/2,791 (0.6\%) high-risk prenatal cases, and 46/3,588 (1.2\%) postnatal cases. The difference was statistically significant $(P<0.005)$.

\section{Low-penetrance CNV syndromes}

Low-penetrance CNVs were detected in 85/15,215 (0.6\%) control cases (low-risk prenatal), 25/2,791 (0.9\%) high-risk prenatal cases, and $35 / 3,588(1.0 \%)$ in postnatal cases. The difference was not statistically significant $(P=0.57)$.

\section{Sex bias}

High-penetrance CNVs (all autosomes) were found in 139 cases (79 males and 60 females). However, the ratio of males to females was significantly higher only in the postnatal group (58/79 (73\%) versus 34/60 (56\%); $P<0.05)$.

\section{DISCUSSION}

The current study focused on the frequency of 20 recurrent CNV syndromes. ${ }^{10,13}$ We analyzed CMA results from two laboratories that performed 21,594 CMA tests in similar populations. These CNVs were detected in 399 cases (1.8\%): $1.0 \%(153 / 15,215)$ in group I (low-risk prenatally that served as a control group), $2.5 \%(70 / 2,791)$ in group II (high-risk prenatally due to malformations detected with ultrasonography or magnetic resonance imaging) and $4.8 \%(173 / 3,588)$ in group III (CMA performed postnatally).

High-penetrance CNVs occurred at a ratio of 1:40 (2.6\%) for postnatal findings compared to $1: 111(0.9 \%)$ in high-risk pregnancies and $1: 1,000(0.1 \%)$ in low prenatal risk cases $(P<0.001)$. Compared to low-risk pregnancies, the frequencies of high-penetrance CNVs were 25-fold in postnatal testing and 9-fold in high-risk pregnancies. Analyzing moderate-penetrance CNVs showed a similar pattern, although with lower absolute values (1.3\% postnatally, $0.7 \%$ high-risk, and $0.3 \%$ in low-risk prenatal cases; $P<0.05$ ). The frequency of low-penetrance CNVs in postnatal testing was $1.0 \%$, in high-risk pregnancies $0.9 \%$, and in low-risk pregnancies $0.6 \%$. Hence, this group did not exhibit the same pattern and no statistical difference was found between groups. This observation suggests that low-penetrance CNVs define a different group and probably do not cause developmental delay/intellectual disability, autism spectrum disorders, or multiple congenital anomalies by themselves. CNV studies are important for several reasons: (i) CNVs have a central role in the etiology of intellectual disability and neurodevelopmental problems, ${ }^{11,12,15}$ (ii) CMA is becoming widespread in the prenatal setting, ${ }^{2}$ (iii) CNVs might interact with point mutations with compound heterozygous genotypes, ${ }^{16}$ and (iv) CNVs might interact with additional CNVs, resulting in a phenotype different than either $\mathrm{CNV}$ alone. ${ }^{4} \mathrm{~A}$ "second-hit" model was proposed based on the observation that affected persons with a microdeletion on chromosome $16 \mathrm{p} 12.1$ are more likely to have more large CNVs than controls do. ${ }^{17}$ Of note is the fact that the segment of chromosome $16 \mathrm{p} 11.2$ - the region of the gene $S H 2 B 1$ that is covered by 57 probes and deletions or duplications-is well detected by our system. One possible explanation for the relatively low numbers detected in two large laboratories compared to other databases ${ }^{18}$ might be a different population profile.

Our observations suggest that high-penetrance CNVs that have a major contribution to the overall heritability of developmental disorders are a more frequent cause of postnatal phenotype, and their representation in the prenatal setting is low. Analyzing specific CNVs in the high-penetrance category found a significant difference between group II and group III CNVs 2, 4, and 5, whereas CNVs 6, 7, and 8 did not show such a difference (Supplementary Table S4). A plausible explanation may be due to the fact that CNVs 6, 7, and 8 are associated with abnormal findings in imaging of the fetus ultrasound and magnetic resonance imaging. No such association exists in CNVs 2, 4, and 5. Low-penetrance CNVs are found in similar frequencies in all groups and are not overexpressed in males. This observation is in accordance with previous reports that found more boys with genomic disorders. These results led the authors to believe that females are less vulnerable to the effects of large variations than are males. This would explain the observation of an increased prevalence of neurodevelopmental disorders among males compared to females. ${ }^{4}$ Thus, we suggest that low-penetrance CNVs detected in low-risk pregnancies should not be considered pathogenic and should not be used as phenotype predictors. They could act in concert with other genetic factors or as part of a multifactorial pattern of inheritance combined with environmental events, but their influence on phenotype cannot be predicted prenatally. Although 15q11.2 deletion is common, we do not think that this CNV has clinical impact that is characterized by causeresult relationship. We feel that this $\mathrm{CNV}$ increased selection bias and activates mainly through multifactorial mode of inheritance. Consequently, a prenatal report of a lowpenetrance CNVs could create unfounded concerns. A study that found that penetrance may be quite different when looking at the effect of a genetic variation detected as part of a screening test in the general population (in our study represented by lowrisk prenatal samples) compared to individuals who were evaluated postnatally due to a personal or strong family history of a neurological disorder ${ }^{19}$ supports these conclusions. CNVs with moderate penetrance should be treated as a separate group. Their associated increased frequency in the postnatal group compared to low-penetrance CNV cases needs to be considered in cautious counseling, especially in the prenatal setting.

A limitation of the current study is that the control group comprised low-risk pregnancies that underwent CMA due to maternal request. The outcome of these pregnancies was not known in most cases. In addition, we evaluated only 20 common syndromes in our cohorts and did not analyze the rest.

In conclusion, low-penetrance $\mathrm{CNV}$ syndromes are not by themselves a cause of intellectual disability or congenital 
anomalies. Thus, we suggest they should be classified as lowpenetrance recurrent CNVs of unclear clinical significance, not otherwise specified. We recommend they should not be reported to the parents. CNVs with moderate penetrance constitute a unique group, found with increased frequency among individuals with developmental delay/intellectual disability, autism spectrum disorders, or multiple congenital anomalies, but definitely to a lesser extent than highpenetrance CNVs are. Prenatal counseling in these cases is expected to be complicated. The parents' CNVs status does not provide complete information about the expected postnatal phenotype because these CNVs are associated with syndromes with partial penetrance and variable clinical expression. The population frequency data are more informative. The frequency of pathogenic CNVs with different penetrance levels in pre- and postnatal CMA might assist couples and genetic counselors who are considering this test during pregnancy. Once recurrent $\mathrm{CNV}$ s are detected by CMA, the penetrance information and perhaps the fetal sex should be factors in genetic counseling.

\section{SUPPLEMENTARY MATERIAL}

Supplementary material is linked to the online version of the paper at http://www.nature.com/gim

\section{ACKNOWLEDGMENTS}

This study was partially supported by the Adler Chair in Pediatrics, Tel Aviv University, Tel Aviv, Israel. The authors thank Faye Schreiber for editing the manuscript and Nava Jelin for the statistical analysis.

\section{DISCLOSURE}

The authors declare no conflict of interest.

\section{REFERENCES}

1. Miller DT, Adam MP, Aradhya S, et al. Consensus statement: chromosomal microarray is a first-tier clinical diagnostic test for individuals with developmental disabilities or congenital anomalies. Am J Hum Genet 2010;86:749-764
2. Wapner RJ, Martin CL, Levy B, et al. Chromosomal microarray versus karyotyping for prenatal diagnosis. N Engl J Med 2012;367:2175-2184.

3. Ben-Shachar S, Lanpher B, German JR et al. Microdeletion 15q13.3: a locus with incomplete penetrance for autism, mental retardation, and psychiatric disorders. J Med Genet 2009;46:382-388.

4. Girirajan S, Rosenfeld JA, Coe BP, et al. Phenotypic heterogeneity of genomic disorders and rare copy-number variants. N Engl I Med 2012; 367:1321-1331.

5. Todarello G, Feng N, Kolachana BS, et al. Incomplete penetrance of NRXN1 deletions in families with schizophrenia. Schizophr Res 2014; 155:1-7

6. Riley, KN, Catalano LM, Bernat JA, et al. Recurrent deletions and duplications of chromosome 2 q11.2 and 2 q13 are associated with variable outcomes. Am J Med Genet 2015;167A:2664-2673.

7. Girirajan S, Eichler EE. Phenotypic variability and genetic susceptibility to genomic disorders. Hum Mol Genet 2010;19:R176-R187.

8. Cooper DN, Krawczak M, Polychronakos C, et al. Where genotype is not predictive of phenotype: towards an understanding of the molecular basis of reduced penetrance in human inherited disease. Hum Genet 2013;132,1077-1130.

9. Beaudet AL. Ethical issues raised by common copy number variants and single nucleotide polymorphisms of certain and uncertain significance in general medical practice. Genome Med 2010;2:42.

10. Rosenfeld A, Coe BP, Eichler EE, et al. Estimates of penetrance for recurrent pathogenic copy-number variations. Genet Med 2013;15: 478-481.

11. Stefansson $H$, Rujescu $D$, Cichon $S$, et al. Large recurrent microdeletions associated with schizophrenia. Nature 2008;455:232-236.

12. Malhotra D, McCarthy S, Michaelson JJ, et al. High frequencies of de novo CNVs in bipolar disorder and schizophrenia. Neuron 2011:72: 951-963.

13. Vassos E, Collier DA, Holden S, et al. Penetrance for copy number variants associated with schizophrenia. Hum Mol Genet 2010;19:3477-3481.

14. Benn PA. Prenatal counseling and the detection of copy-number variants. Genet Med 2013;15:316-317.

15. Allen AS, Berkovic SF, Cossette $P$, et al. Epi4K Consortium; Epilepsy Phenome/Genome Project: de novo mutations in epileptic encephalopathies. Nature 2013;501:217-221.

16. Albers CA, Paul DS, Schulze $H$, et al. Compound inheritance of a lowfrequency regulatory SNP and a rare null mutation in exon-junction complex subunit RBM8A causes TAR syndrome. Nat Genet 2012;44: 435-439, S1-2.

17. Girirajan S, Rosenfeld JA, Cooper GM, et al. A recurrent $16 \mathrm{p} 12.1$ microdeletion supports a two-hit model for severe developmental delay. Nat Genet 2010;42:203-209.

18. Coe BP, Witherspoon K, Rosenfeld JA, et al. Refining analyses of copy number variation identifies specific genes associated with developmental delay. Nat Genet 2014;46:1063-71.

19. Schaaf CP, Wiszniewska J, Beaudet AL. Copy number and SNP arrays in clinical diagnostics. Annu Rev Genomics Hum Genet 2011;12:25-51. 